\title{
Two Stage Hip Revision in Periprosthetic Infection: Results of 41 Cases
}

\author{
Giovanni Pignatti ${ }^{1}$, Shingo Nitta ${ }^{2}$, Nicola Rani ${ }^{*}{ }^{1}$, Dante Dallari ${ }^{1}$, Giacomo Sabbioni ${ }^{1}$, \\ Cesare Stagni ${ }^{1}$ and Armando Giunti ${ }^{1}$ \\ ${ }^{1} 7^{\text {th }}$ Ward of Orthopedic-Trauma Surgery, Rizzoli Orthopedic Institute, Bologna, Italy \\ ${ }^{2}$ Department of Orthopedic Surgery, Hyogo Prefectural Amagasaki Hospital, Hyogo, Japan
}

\begin{abstract}
Background: two-stage revision is considered the best treatment approach for the eradication of chronic joint infection. We report the outcome of 41 consecutive patients with infected hip prostheses, treated between 2000 and 2005 , with two-stage revision using an antibiotic-loaded cement spacer.

Methods: Patients underwent a treatment protocol which included clinical and radiographic evaluation, laboratory investigations, hip aspiration, 99mTc-MDP and 99mTc-leukocyte-labeled scintigraphy and intraoperative assessment. All patients were diagnosed with a late chronic infection and classified as B-host according to the Cierny-Mader classification system. 9 patients out of $41(22 \%)$ required a second interim treatment period, with exchange of the spacer. The proportion of methicillin-resistant Staphylococcus was similar between the one-spacer group and two-spacer group (28\% vs 33\%), whereas the proportion of patients with three or more risk factors was significantly higher in the two-spacer group than in the one-spacer group ( $28 \%$ vs $55 \%$, respectively).
\end{abstract}

Results: Forty patients had final reimplantation, one patient had a resection arthroplasty. At an average follow-up of 5.3 years no recurrence of infection occurred. The average post-operative Harris hip score improved from 41 to 80 .

Conclusions: In the treatment of two-stage revision arthroplasty the adherence to the protocol proved to be effective for infection eradication and final reimplantation.

Keywords: Infection, hip, revision, spacer.

\section{INTRODUCTION}

Deep periprosthetic infection in arthroplasty surgery is a serious problem and its treatment requires considerable effort and cost. Several treatment options have been reported: long-term antibiotic suppression [1, 2], resection (Girdlestone) arthroplasty [2-5], debridement with prosthesis retention [6], one-stage revision [7], and two-stage revision with antibiotic-loaded cement beads or spacer [8], or with spacer prosthesis [9].

It is generally well accepted that it is impossible to eradicate chronic implant-associated infections without removing the foreign bodies [6]. This implies that the surgical options such as resection arthroplasty, one and twostage revision arthroplasty have the potential to eradicate infection.

However, resection arthroplasty, even if used as a temporary method between stages, is associated with a considerable loss of function, and should be considered in patients unfit to have an additional reconstructive procedure and non cooperative patients. Also a severe deficiency of bone stock and poor quality of local soft tissue are indications for an excision arthroplasty $[10,11]$. In these cases, the eradication rate is not very high [12].

*Address correspondence to this author at the $7^{\text {th }}$ Ward of OrthopedicTrauma Surgery, Rizzoli Orthopedic Institute, via Pupilli 1, 40136 Bologna, Italy; Tel: +390516366437; Fax: +390516366840;

E-mail: nicola.rani@tiscali.it
One-stage revision has the advantage of a low treatment cost and preservation of patient function as no interim period is required. In this procedure, pre-operative detection of the infecting pathogen is mandatory and bone cement loaded with antibiotics chosen according to the pathogen sensitivity must be used for prosthesis fixation. A concern remains about mid- and long-term results of cemented revision arthroplasty because of poor cement interdigitation $[13,14]$. In particular reconstruction is difficult in patients with severe bone loss. In case of recurrence, re-revision surgery can be more complicated because of the difficulty of removing the well fixed prosthesis and cement. The eradication rate of one-stage revision arthroplasty compared to two-stage revision arthroplasty has been reported to be lower [7].

With the above in mind, in the last years two-stage revision has become our standard therapeutic approach, not only for the higher eradication rate reported, but also for the increasing percentage of resistant pathogens [15, 17]. Twostage revision consists of a two-step procedure: removal of infected prosthesis, cement, infected and devitalized tissue, and implantation of a considerable number of cement beads or a cement spacer. After 8-12 weeks, during which a systemic antibiotic therapy is given, the second step is carried out (following normalization of the serological markers of infection) which consists of removal of the cement beads or spacer, and implantation of a new prosthesis.

The possibility exists that the prosthesis which is explanted during the first operation, can be used as spacer 
after resterilisation. Few cases are described in literature for the treatment of knee periprosthetic infections $[9,16]$. Even if authors report good results, this technique is not very diffused because of the risk that prosthetic spacers serve as good substrate for bacterial adherence.

Even though initially resection arthroplasty or antibioticloaded cement beads were used in the interim period, these options did not allow maintenance of patient function and leg length. Moreover, the second stage required significant effort for the surgeon because of adhesions and soft tissue contracture around the pathologic hip. To address this problem, many surgeons shifted to the use of a temporary articulating cement spacer.

Many studies have reported good results using cement spacers, both in terms of function in the interim period and also fewer complications after the second operation [18-21]. Therefore, two-stage revision with an antibiotic-loaded cement spacer has become the gold-standard for the treatment of infected hip prostheses [22]. Articulated spacers may be used in this interim period. Recently, several reports about the articulated spacer PROSTALAC (prosthesis of antibiotic-loaded acrylic cement) have shown good results $[10-18,23]$. This system, available only in North America, is a THA design spacer consisting of a thin polyethylene acetabular liner and a femoral stem covered by antibioticloaded cement.

More recently, industrially preformed spacers (Spacer$\mathrm{G}^{1}$ ) in different sizes have become available worldwide, and good results have been reported [24-26]. Protocols for the treatment of an infected hip arthroplasty may be difficult to carry out as planned, because they depend on several factors, such as patient general condition, number and type of pathogens, sensitivity to antibiotics, infection extent. Furthermore, infections caused by an antibiotic-resistant organism have increased [15] making this procedure even more difficult. Even if the infection is deemed eradicated before the second stage operation, infection may be identified at the time of the second stage operation or after the operation, when the results of intraoperative cultures become available.

The purpose of this study is to report the treatment outcome of deep periprosthetic infections treated with twostage revision arthroplasty using preformed antibiotic-loaded spacers, and to evaluate the effectiveness of the treatment method and our protocol.

\section{MATERIAL AND METHODS}

We retrospectively reviewed the treatment outcome of 41 consecutive patients (41 hips) with infected hip prostheses treated with two-stage revision with antibiotic-loaded cement spacers in our institution between 2000 and 2005. Sixteen patients were men and 25 were women. The average age of the patients at first stage surgery was 59 years (range, 28-79 years).

Periprosthetic infection was diagnosed through clinical and radiographic evaluation, simple laboratory investigations (erythrocyte sedimentation rate, ESR; C-reactive protein,

\footnotetext{
${ }^{1}$ Spacer-G is distributed in US with the brand name InterSpace ${ }^{\circledR}$ for Hip by
} Exactech Inc., Gainesville, FL, USA.
CRP; white blood cell, WBC) hip aspiration, ${ }^{99 \mathrm{~m}} \mathrm{Tc}-\mathrm{MDP}$ and ${ }^{99 \mathrm{~m}} \mathrm{Tc}$-leukocyte-labeled scintigraphy and intra-operative assessment.

The ${ }^{99 \mathrm{~m}} \mathrm{Tc}-\mathrm{MDP}$ and ${ }^{99 \mathrm{~m}} \mathrm{Tc}-$ leukocyte-labeled scintigraphies were performed in all patients except in those cases with clear evidence of infection, such as the presence of a fistula. A sample for culture was taken either from the fistula or through hip aspiration in order to isolate the organism and to be aware of its sensitivity and possible resistance to antibiotics before the first stage.

The pre-operative clinical evaluation included general health status, patient history, pain and wound condition. Preoperative plain radiographs included anterior-posterior view of the pelvis and lateral view of the hip joint were obtained to facilitate the radiographic evaluation of evidence of loosening and migration, or presence of a hidden sinus.

As patient's individual risk factors influence treatment options, prognosis and interpretation of treatment results, we considered the physiological classification proposed by Cierny-Mader [27]: A-hosts are healthy and without healing deficiencies; B-hosts are compromised by one or more local and/or systemic comorbidities; C-hosts a host for whom the morbidity of treatment is worse than that imposed by the disease itself: C-hosts are not considered candidates for aggressive surgical intervention.

In the first stage operation we performed a thorough debridement, removal of all foreign bodies (prosthesis, plug, cement and so on) and potentially infected soft tissue. All operations were performed through a lateral approach. When it was difficult to remove the foreign bodies, extended trochanteric osteotomies were performed without hesitation. When a sinus was present Methylene Blue was injected in order to visualize all the potentially infected soft tissue.

Prior to administration of antibiotics and debridement at least three samples were taken for culture, from joint capsule, acetabulum, and femoral canal. All surgical fields were irrigated with a pulsed lavage system thoroughly. Both the acetabulum and femoral canal were reamed in order to remove all potentially infected and necrotic tissues and implant the antibiotic loaded cement spacer.

We used Spacer-G (Tecres S.p.A. Sommacampagna, Verona, Italy) in 36 cases and the StageOne ${ }^{\mathrm{TM}}$ Cement Spacer Mold (Biomet ${ }^{\mathbb{B}}$ Orthopaedics Inc., Warsaw, Indiana, USA) in 5 cases. Both types of cement spacers have the shape of a unipolar hemiarthroplasty.

Spacer-G is an industrially preformed device (Fig. 1) composed by a main structure in stainless steel coated with bone cement (PMMA) with gentamicin, and is available in 3 head sizes (46-54-60mm), and 2 stem lengths (short and long), while the StageOne ${ }^{\mathrm{TM}}$ Cement Spacer Mold is a silicone mould (Fig. 2) in four different head sizes (43-51$57-64 \mathrm{~mm}$ ), which has to be prepared intra-operatively with bone cement and antibiotics. The type of spacer was selected according to the sensivity of the antibiogram, and also according to bone loss and acetabulum size.

When Spacer-G was used, a proximal neck cementation was always performed to improve or achieve stability. Cement was always loaded with Vancomycin (4gr added to each $40 \mathrm{gr}$ polymer powder prior to curing the cement). 

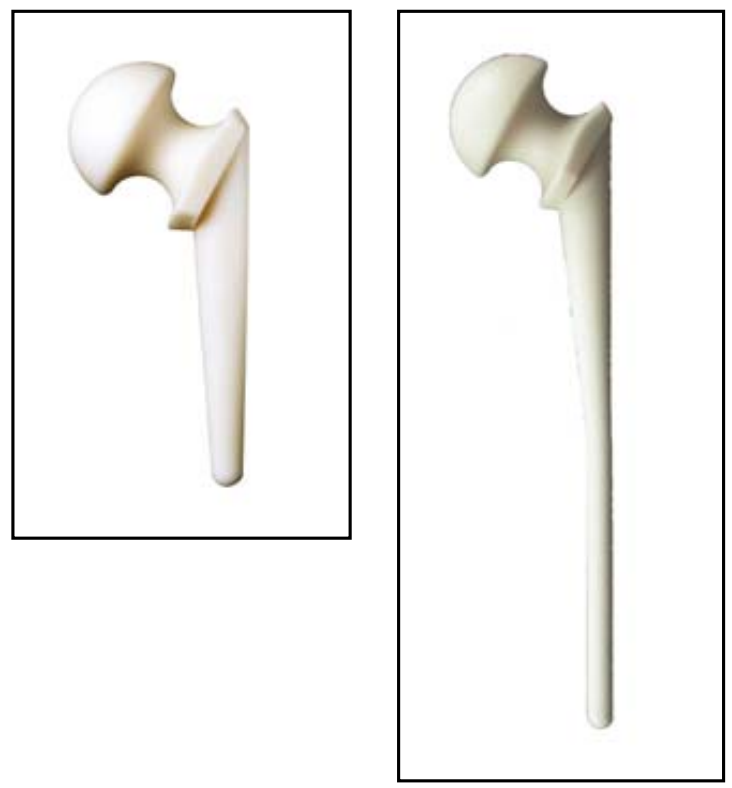

Fig. (1). Standard and long stem Spacer-G.

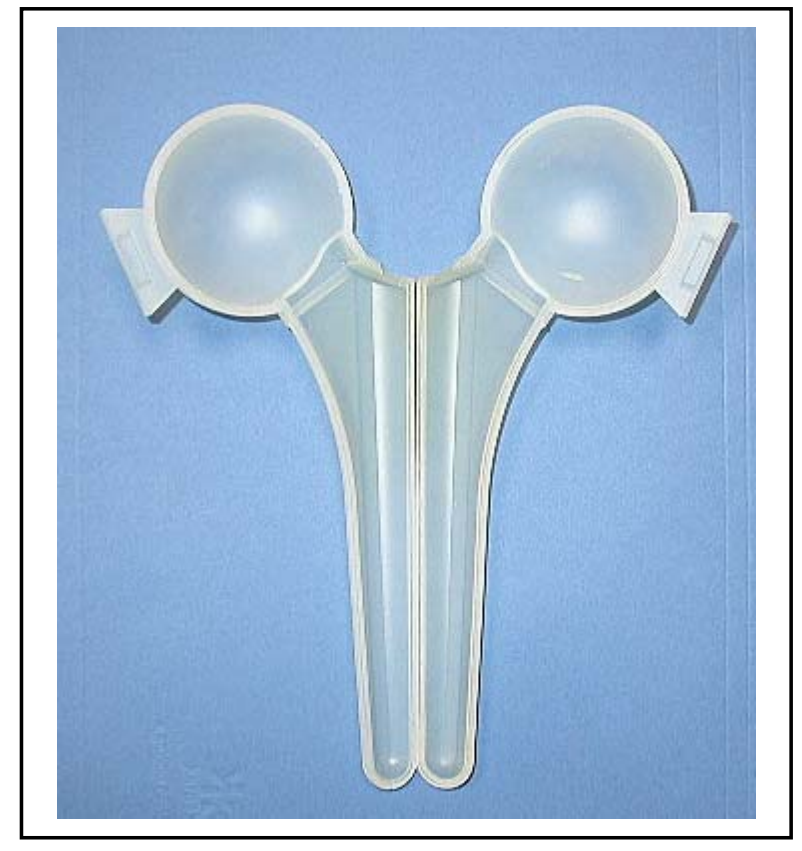

Fig. (2). StageOne ${ }^{\mathrm{TM}}$ cement spacer mould.

When the StageOne ${ }^{\mathrm{TM}}$ Cement Spacer Mold was used, cement was loaded with Vancomycin (4gr each $40 \mathrm{gr}$ cement) or Vancomycin and Meropenem $(2 \mathrm{gr}+2 \mathrm{gr}$ each $40 \mathrm{gr}$ cement). Additionally one or two Kirschner wires were inserted as an endoskeleton, and antibiotic loaded cement was injected into the mold in a doughy state.

At the end of the first operation two suction drain tubes were inserted in the hip joint.

The interim period included six weeks of antibiotic therapy and subsequently at least one week of antibiotic discontinuation. Antibiotic therapy was chosen in collaboration with the infectious disease specialist, if necessary. General and local conditions and laboratory tests were checked regularly. After the removal of the drain tubes on the second PO day, all patients were allowed to walk with touch-down weight bearing. The criteria for the second stage operation included negative ESR and CRP or gradual improvement of laboratory values, negative $99 \mathrm{mTc}$ leukocyte-labeled scan, and negative hip joint aspiration. If the patients shifted from B-host to C-host during the interim period, a resection arthroplasty was performed.

In the second stage operation the spacer was removed and at least three samples were taken for culture and antibiotic sensitivity, followed by additional debridement using the lavage system.

Revision hip arthroplasty was performed using structural or morcellized allograft when bone loss was severe. Postoperative rehabilitation based on the stability of the implants and the patient's status was carried out.

Administration of prophylactic antibiotics was continued until the intra-operative samples were proven to be negative. If the results of the intra-operative samples were positive despite pre-operatively negative results, a targeted antibiotic therapy was administered for 2 weeks. If the results of the samples were negative, the patients were followed-up without antibiotic administration. If the results of laboratory tests were abnormal prior to the second stage operation, or if the leukocyte labeled bone scan or hip aspiration were positive, a thorough debridement was performed and the cement spacer was exchanged with a new one, and subsequently the treatment protocol of a second interim period was carried out till reimplantation.

According to the number of interim periods, all patients were divided into one-spacer and two-spacer groups.

The patients regularly underwent clinical, radiographic and laboratory tests. The functional outcome was evaluated before the first stage operation and at the time of the latest follow-up using the Harris Hip Score [26].

The incorporation between allograft and host bone was examined by comparing the immediate post-operative X-ray with the latest one.

\section{RESULTS}

In the present study all patients followed our treatment protocol (Fig. 3).

\section{Pre-Operative Assessment}

The main clinical symptom was pain in all patients. CRP and/or ESR were pre-operatively elevated in most patients. A discharge from the fistula was seen in 11 patients, all had a culture made, but in only 4 was the organism identified. Hip aspiration was performed in the remaining 30 patients: in 24 patients the organism was identified. Most organisms identified before the first operation were Staphylococcus species $(51 \%)$. Leukocyte-labeled scintigraphy was positive in 32 patients. Patients with a negative scan had a positive hip aspiration or elevated laboratory indexes.

The mean time lapse between the previous hip arthroplasty and the infection onset symptoms was 41.4 


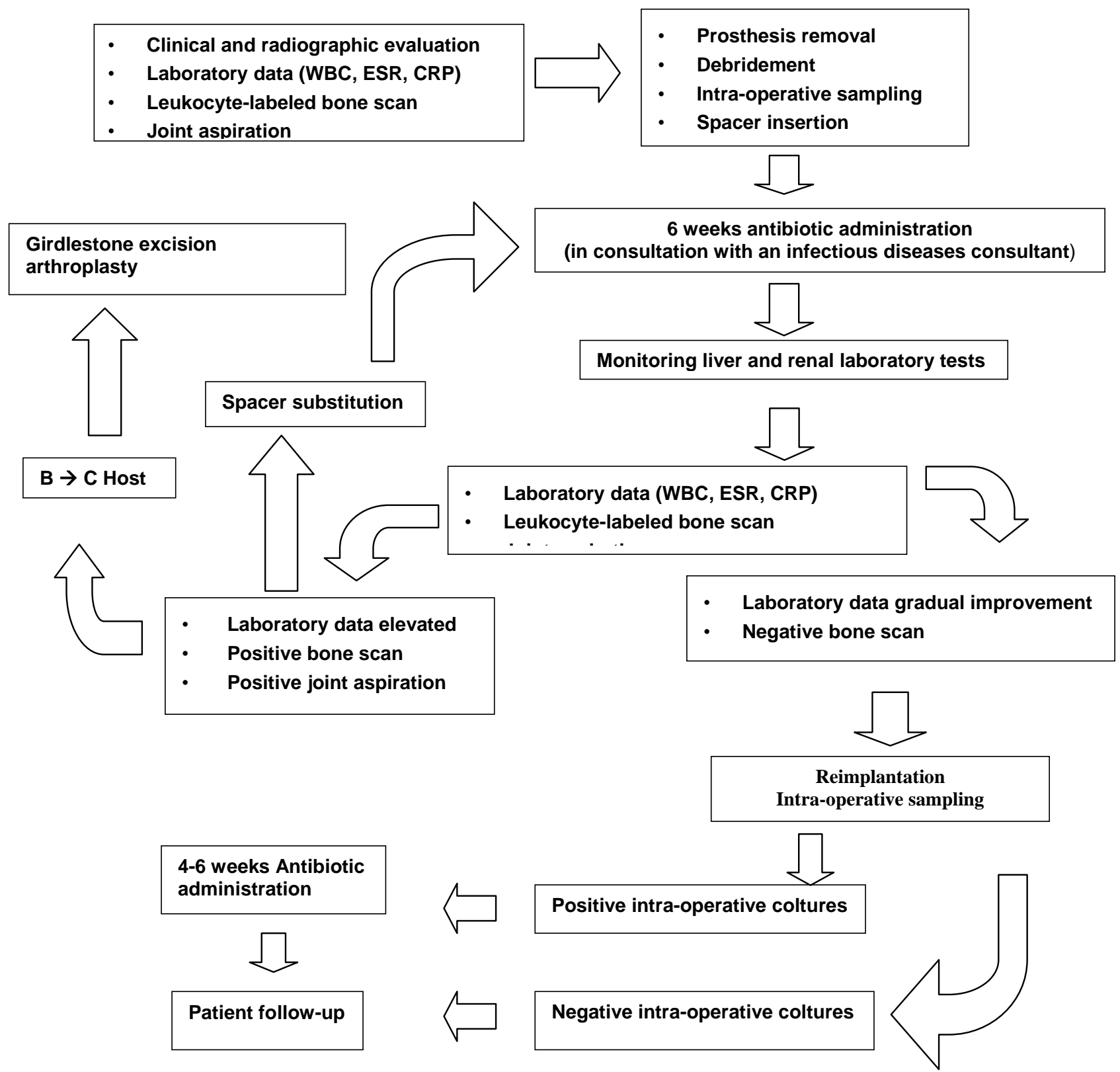

Fig. (3). Treatment protocol for infected hip arthroplasty.

months (range, 1.5-114) months. According to the classification of Tsukayama et al. [6], all patients were diagnosed with a late chronic infection.

Pre-operatively all patients were classified as B-host according to Cierny-Mader classification [27]. Three or more morbidities were present in 14 patients $(36 \%)$.

\section{The First Operation and the Interim Period}

The short-stem Spacer-G was used in 29 patients, and the long-stem was used in 7 patients. In all cases the neck was cemented with vancomycin-loaded bone cement $(4 \mathrm{~g}$ x $40 \mathrm{~g}$ PMMA). StageOne ${ }^{\mathrm{TM}}$ Cement Spacer Mold was used in 5 patients: in 3 patients bone cement was loaded with Vancomycin and Meropenem (2gr $+2 \mathrm{gr}$ added to each $40 \mathrm{gr}$ polymer powder prior to curing the cement), in the remaining 2 patients only Vancomycin (4gr per 40gr pack) was used. In one case the Cement Spacer Mold broke 6 days after the first operation, and so the spacer was exchanged with a new one. This case was included in the two-spacer group.

Infection was caused mainly by Staphylococcus species (Table 1). Methicillin-resistant Staphylococcus was identified in 12 of 41 patients $(29.5 \%)$. Multiple organisms were identified in 10 patients. In only one patient the intraoperative organism (Staphylococcus epidermidis) was different from the pre-operative organism (Enterobacter spp.).

After the first operation all 41 patients were regularly evaluated with clinical and laboratory findings. A ${ }^{99 \mathrm{~m}} \mathrm{Tc}$ leukocyte labeled scan was performed in all patients at least 7 days after antibiotic treatment had been completed. Hip 
aspiration was performed in 29 patients due to suspected infection and was negative in 21 patients. Of the 8 patients with positive culture, scintigraphy was negative in 5 and positive in 3 . The 12 patients without hip aspiration had a good clinical status, normal laboratory values, and negative scintigraphy. Therefore, these patients were considered free of infection. Only one patient still had the symptom of persistent infection after antibiotic therapy in the interim period. Thirty-two patients (78\%) fulfilled the criteria for reimplantation (one-spacer group). One (11\%) of 9 patients with at least one positive result shifted from B-host to C-host in the interim period. This patient underwent a resection arthroplasty (Girdlestone), and the infection was eventually eradicated. As a result, the treatment protocol of a second interim period was performed in the remaining 8 patients (two-spacer group). In one of these patients it was necessary to evacuate an abscess in the ipsilateral iliac fossa.

Table 1. Organisms cultured in the First Operation

\begin{tabular}{|l|l|}
\hline Staphylococcus epidermidis (methicillin resistant) & $\mathbf{1 3}(\mathbf{5})$ \\
\hline Staphylococcus aureus (methicillin resistant) & $\mathbf{1 1}(\mathbf{4})$ \\
\hline Enterococcus & $\mathbf{2}$ \\
\hline Streptococcus & $\mathbf{1}$ \\
\hline Polymicrobial flora & $\mathbf{1 0}$ \\
\hline Not identified & $\mathbf{4}$ \\
\hline
\end{tabular}

In nine $(28 \%)$ of the 32 patients with one cement spacer (one-spacer group), and in three (33\%) of the 9 patients with two cement spacers (two-spacer group), the causative organism was methicillin-resistant Staphylococcus. There was no significant difference between the one-spacer group and two-spacer group regarding the ratio of methicillinresistant Staphylococcus. There were three or more morbidities in five $(55 \%)$ of 9 patients in the two-spacer group compared to nine $(28 \%)$ of 32 patients in the onespacer group. The patients' morbidities were higher in the two-spacer group than in the one-spacer group.

\section{The Second Operation}

Forty patients, except for one patient with a Girdlestone arthroplasty, underwent revision hip arthroplasty using 40 acetabular cementless cups, 34 cementless stems and six cemented stems. Bone allografts were used in five cases. At least three intra-operative cultures were taken in all 40 hips. The result of the three cultures showed that no organism was present in 25 hips, one of the three samples was positive in 10 hips, two of the three samples were positive in two hips, and all three samples were positive in three hips. The organism was Staphylococcus epidermidis in 14 hips and Staphylococcus aureus in one hip. All staphylococcus species were sensitive to methicillin. The 15 patients with positive results were administered antibiogram-tailored antibiotic treatment for four to six weeks. After the operation, all patients were regularly followed-up with clinical evaluation and laboratory tests. After a mean followup of 5.3 years (range 4-9) no recurrence of infection has been detected.
The average post-operative Harris hip score improved from 41 to 80 .

\section{Complications}

Complications in the interim period included two dislocations and one breakage of the cement spacer (one dislocation with the Spacer-G, and one dislocation and one breakage with the StageOne ${ }^{\mathrm{TM}}$ Cement Spacer Mold). Both dislocations were treated by surgical reduction. The breakage of the spacer was treated by exchanging it with a new one.

After the second stage operation, two patients had a dislocation of the revision prosthesis. One was managed with conservative therapy and one was treated with a revision of the acetabular cup. Other complications included one aseptic loosening of the cementless stem, treated with stem revision surgery, and one post-operative arterial bleeding, treated with embolization.

The 2 patients with loosening of the acetabular cup and cementless stem during the follow-up period had at least three intra-operative samples taken during the revision surgery. No organism was found in the revision stem, but $S$. epidermidis was isolated in the revision cup in one sample: the patient received antibiotic therapy for 2 weeks, and had no symptoms of recurrence of the infection.

\section{Radiographic Evaluation}

As mentioned above, two patients had an aseptic loosening (one had the loosening of the cup and the other had the loosening of the cementless stem). All acetabular and femoral components, except for these two, appeared to be radiographically stable at the latest observation. All five allografts used for the patients with severe bone deficiency appeared to have integrated with host bone.

\section{DISCUSSION}

The results of our treatment protocol are satisfactory. All deep periprosthetic infections were eradicated, and forty of 41 patients had final reimplantation.

Nowadays, two-stage revision arthroplasty is considered the gold standard treatment method for deep periprosthetic infection [22], however it involves a high cost for the medical healthcare system, and much effort for patients, paramedical staffs and surgeons. It needs at least two operations to cure. In contrast, one-stage revision arthroplasty has the advantage of a lower cost and function preservation. The success of one-stage revision is highly associated with the correct choice of the specific antibiotics added to the bone cement used for prosthesis fixation. Hanssen and Rand [29] reported a success rate of $83 \%$ with antibiotic-loaded cement and a success rate of $60 \%$ without antibiotic-loaded cemen; Raut et al. [30, 31] reported a success rate of $86 \%$ in 57 hips with draining sinuses at an average follow-up of 7.4 years, and a success rate of $93.4 \%$ in patients with gram-negative infections at an average follow-up of 8 years. However, other authors [15, 32, 33] reported that the eradication rate in one-stage revision arthroplasty was lower than in two-stage revision arthroplasty when highly virulent organisms such as gram negative organisms, coagulase- negative staphylococci, and 
anaerobic micro-organisms were causing the infection. Therefore, it has been proposed that the organism should be of lower virulence and antibiotic-loaded cement should be used when performing one-stage revision arthroplasty [3537]. In addition one-stage revision in patients with severe bone loss remains a concern due to the poor mid- or longterm results of cemented revision arthroplasty [13, 14].

The diagnosis of deep periprosthetic infection is a challenging problem. There is no $100 \%$ sensitive and specific test, and before the final reimplantation, many surgeons may have to face the difficult situation in which there is no certainty that infection has been eradicated. In our study all patients were managed with two-stage revision arthroplasty using a same protocol. In the interim period, the protocol included regular laboratory tests, hip aspiration, ${ }^{99 m}$ Tc-leukocyte labeled scintigraphy, and the physiological stage Cierny-Mader classification.

To our knowledge, there are only a few reports which have dealt with deep periprosthetic infections taking into consideration the patient risk factors. Cierny et al. [38] reported the treatment result of 43 patients with periprosthetic infection, using an osteomyelitis classification system designed to stratify treatment selection according to patient risk factors. In this study the implant survival rate of the patients with three or more morbidities was $0 \%$ at two years follow-up. They concluded that the infection duration and the condition of the host are the two most important variables in predicting outcomes in patients with prosthetic infections.

Although $14(36 \%)$ of 41 patients were B-host with three or more morbidities in our study, a final reimplantation was performed in 13 and a resection arthroplasty was performed only in one due to the worsening of the patient's condition. In the 14 high-risk patients, no recurrence of the infection occurred up to their last follow-up visit.

Deep periprosthetic infection caused by resistant organisms has been increasing over recent decades, and surgery has become more and more complicated compared to infection caused by non-resistant organisms. Kilgus et al. [39] reported the outcome of 70 deep hip and knee periprosthetic infections and compared the results of patients infected with resistant organisms and non-resistant organisms. They found that the eradication rate of the former group was much lower compared to the latter group in which the bacterial strains were sensitive to at least one antibiotic. In contrast, Volin et al. [40] compared the outcomes of two groups of patients (a methicillin-sensitive group and a methicillin-resistant group) treated only with two-stage revision arthroplasty, and reported that the efficacy of twostage reconstruction was similar in both groups.

In our study, 12 of 41 patients $(29.5 \%)$ had a methicillinresistant Staphylococcus, and we did not observe any difference between the one-spacer group and the two-spacer group which had a similar methicillin-resistant proportion (28 and 33\%, respectively). However, the proportion of patients with three or more risk factors was higher in the two-spacer group than in the one-spacer group (55 vs $28 \%$, respectively). Our results demonstrate that it is more difficult to eradicate the infection in the patients with three or more risk factors.
In our opinion two-stage revision is more versatile for the strategy of infection eradication and for the choice of the type of reconstruction compared to one-stage revision. First, two-stage revision allows the assessment of response to the antibiotics selected. If the organism detected pre-operatively is different from the organism detected in the first stage, it is possible to change the antibiotic treatment. Second, the method allows reconstruction using either cementless or cemented components. Although the final reimplantation has traditionally been performed with cemented components and antibiotic-loaded cement, recently many authors [23, 41, 42] have recommended cementless reimplantation and have reported encouraging results not only following aseptic [43], but also septic loosening of hip arthroplasty.

In the present study cementless components were used in all acetabula and in 34 of 40 femurs in the final reimplantation. One cementless acetabular component and one cementless femoral component became loose postoperatively, and were revised. As a result, all components, including the two newly revised components, appeared to be stable radiographically at the latest observation.

Finally, the method allows the use of allograft in patients with severe bone loss. In our study, morselized and bulk allografts were used in five patients with severe bone loss. The drawback is the possibility of infection recurrence: Masri et al. [20] reported that two of the three recurrences in their study had allograft bone in the final reconstruction. In contrast, Berry et al. [44] reported the use of bone allografts to reconstruct deficient acetabular and femoral bone in eighteen patients. At a mean of 5.3 years after reimplantation, only two patients had had recurrence of the infection. Wang and Chen [45] used a combination of morselized and bulk allograft in 22 patients with an eradication rate of $91 \%$. Alexeeff et al. [46] reported 11 patients with massive structural allografts in the final reimplantation of two-stage revision, and no recurrence at four years of follow-up. Similarly, in our study no recurrence occurred in the five patients with allografts at the last followup time, and all allografts appeared to be incorporated in the host bone.

In our study Spacer-G, pre-loaded with gentamicin 1.9\% w/w, was used in most cases and the spacer neck was routinely cemented using $4 \mathrm{~g}$ vancomycin per pack of cement $(10 \% \mathrm{w} / \mathrm{w})$. Compared to many reports, we used a high dose of vancomycin in the cement. It is well-known that use of high-dose antibiotics has the potential of systemic side-effects, such as nephrotoxicity. However, Springer et al. [34] evaluated the safety and potential complications of a high-dose antibiotic-loaded cement spacer for the treatment of an infected total knee arthroplasty treated with two-stage resection arthroplasty. They found that no patients showed any clinical evidence of systemic side-effects of the antibiotics. In our study no disease caused by systemic sideeffects of the antibiotics occurred.

As the result of hip aspiration is shown to be sometimes inaccurate according to several reports [47], we conducted hip aspiration in 29 patients with suspected infection in the interim period. As a result, eight patients with at least one positive result underwent a second interim period. Finally, those patients had a final reimplantation without recurrence of infection until the last visit. Recently, various methods to 
visualize periprosthetic infections using nuclear imaging technique have been described. Pelosi et al. [48] showed that sensitivity and specificity were $95.6 \%$ and $95.8 \%$ respectively using ${ }^{99 \mathrm{~m}} \mathrm{Tc}-\mathrm{HMPAO}-$ leukocyte labeled scintigraphy by means of semiquantitative evaluation. Also Larikka et al. [49] conclude that leucocyte imaging improves the specificity of diagnosis of infected hip prostheses.

There are some limitations to this study. The sample size in this series may be considered small, however for this kind of pathology such a number of patients treated in a single Institution and with a same protocol is a consistent number. Secondly, no control group managed with one-stage revision was present, nonetheless two-stage revision is accepted today as the gold standard in the treatment of deep periprosthetic infection, and the eradication rate achieved stands for it.

In conclusion, there was no recurrence of infection in our study at mean follow-up of 4.3 years. If we consider a failure to be either no device reimplantation or a resection arthroplasty, the success rate of the final reimplantation was $98 \%(40 / 41)$.

This study suggests that the success rate depends on the correct application of a protocol, which considers the patient risk factors and the meticulous evaluation of the different diagnostic tests.

We believe that the use of a standardized protocol for two-stage revision arthroplasty leads to successful eradication of infection and reconstruction.

\section{REFERENCES}

[1] Goulet JA, Pellicci PM, Brause BD, Salvati EM. Prolonged suppression of infection in total hip arthroplasty. J Arthroplasty 1988; 3(2): 109-16.

[2] Canner GC, Steinberg ME, Heppenstall RB, Balderston R. The infected hip after total hip arthroplasty. J Bone Joint Surg Am 1984; 66: 1393-99.

[3] Bourne RB, Hunter GA, Rorabeck CH, Macnab JJ. A six-year follow-up of infected total hip replacements managed by Girdlestone's arthroplasty. J Bone Joint Surg Br 1984; 66: 340-3.

[4] McDonald DJ, Fitzgerald RH Jr, Ilstrup DM. Two-stage reconstruction of a total hip arthroplasty because of infection. J Bone Joint Surg Am 1989; 71: 828-34.

[5] McElwaine JP, Colville J. Excision arthroplasty for infected total hip replacements. J Bone Joint Surg Br 1984; 66: 168-71.

[6] Tsukayama DT, Estrada R, Gustilo RB. Infection after total hip arthroplasty. A study of the treatment of one hundred and six infections. J Bone Joint Surg Am 1996; 78-A(4): 512-23.

[7] Garvin KL, Hanssen AD. Current concepts review. Infection after total hip arthroplasty. Past, present, future. J Bone Joint Surg Am 1995; 7-A(7): 1576-88.

[8] Hsieh PH, Shih CH, Chang YH, Lee MS, Shih HN, Yang WE. Two-stage revision hip arthroplasty for infection: comparison between the interim use of antibiotic-loaded cement beads and a spacer prosthesis. J Bone Joint Surg Am 2004; 86-A(9): 1989-97.

[9] Jämsen E, Sheng P, Halonen P, Lehto, et al. Spacer prostheses in two-stage revision of infected knee arthroplasty. Int Orthop 2006; 30: 257-61.

[10] De Laat EA, van der List JJ, van Horn JR, Sloof TJ. Girdlestone's pseudarthrosis after removal of a total hip prosthesis; a retrospective study of 40 patients. Acta Orthop Belg 1991; 57(2): 109-13.

[11] Lieberman JR, Callaway GH, Salvati EA, Pellicci PM, Brause BD. Treatment of the infected total hip arthroplasty with a two-stage reimplantation protocol. Clin Orthop Relat Res 1994; 301: 205-12.

[12] Nestor BJ, Hanssen AD, Ferrer-Gonzalez R, Fitzgerald RH Jr. The use of porous prostheses in delayed reconstruction of total hip replacements that have failed because of infection. J Bone Joint Surg Am 1994; 76-A: 349-59.

[13] Dohmae Y, Bechtold JE, Sherman RE, Puno RM, Gustilo RB. Reduction in cement-bone interface shear strength between primary and revision arthroplasty. Clin Orthop Relat Res 1988; 236: 21420.

[14] Lie SA, Havelin LI, Furnes ON, Engesaeter LB, Vollset SE. Failure rates of 4762 revision total hip artrhroplasties in the Norwegian Arthroplasty Register. J Bone Joint Surg Br 2004; 86(4): 504-9.

[15] EARRS Annual Report 2006. EARSS The European Antimicrobial Resistance Surveillance System. 2009 February 17. Available from http://www.rivm.nl/earss/

[16] Emerson RH Jr, Muncie M, Tarbox TR, Higgins LL. Comparison of a static with a mobile spacer in total knee infection. Clin Orthop Relat Res 2002; 404: 132-8.

[17] Evans R, Clyburn T, Joseph J, et al. Orthopaedic infection: community-acquired and hospital-acquired methicillin-resistant Staphylococcus aureus (MRSA). AAOS patient safety committee scientific exibit 2008. 2009 February 18. Available from: http://www.aaos.org/research/committee/ptsafety/ptsafety.asp

[18] Younger ASE, Duncan CP, Masri BA, McGraw RW. The outcome of two-stage arthroplasty using a custom-made interval spacer to treat the infected hip. J Arthroplasty 1997; 12(6): 615-23.

[19] Younger ASE, Duncan CP, Masri BA. Treatment of infection with segmental bone loss in the proximal part of the femur in two stages with use of antibiotic-loaded interval prosthesis. J Bone Joint Surg Am 1998; 80-A(1): 60-9.

[20] Masri BA, Duncan CP, Beauchamp CP. Long-term elution of antibiotics from bone-cement: An in vivo study using the prosthesis of antibiotic-loaded acrylic cement (PROSTALAC) system. J Arthroplasty 1998; 13: 331-8.

[21] Haddad FS, Masri BA, Garbuz DS, Duncan CP. The treatment of the infected hip replacement. The complex case. Clin Orthop Relat Res 1999; 369: 144-56.

[22] Anagnostakos K, Fürst O, Kelm J. Antibiotic-impregnated PMMA hip spacers: Current status. Acta Orthop 2006; 77(4): 628-37.

[23] Masri BA, Panagiotopoulos KP, Greidanus NV, Garbuz DS, Duncan CP. Cementless two-stage exchange arthroplasty for infection after total hip arthroplasty. J Arthroplasty 2007; 22: 72-8.

[24] Magnan B, Regis D, Biscaglia R, Bartolozzi P. Preformed acrylic bone cement spacer loaded with antibiotics: use of two-stage procedure in 10 patients because of infected hips after total replacement. Acta Orthop Scand 2001; 72(6): 591-4.

[25] D'Angelo F, Negri L, Zatti G, Grassi FA. Two-stage revision surgery to treat an infected hip implant. A comparison between a custom-made spacer and a pre-formed one. Chir Organi Mov 2005; 90(3): 271-9.

[26] Meani E, Romanò CL, Crosby L, Hofmann G. Infection and local treatment in orthopaedic surgery. Berlin Heidelberg (GER): Springer-Verlag, 2007

[27] Cierny G, Mader JT, Pennick H. A clinical staging system of adult osteomyelitis. Contemp Orthop 1985; 10: 17-37.

[28] Harris WH. Traumatic arthritis of the hip after dislocation and acetabular fractures: treatment by mold arthroplasty. J Bone Joint Surg Am 1969; 51(4): 737-55.

[29] Hanssen AD, Rand JA. Evaluation and treatment of infection at the site of a total hip or knee arthroplasty. J Bone Joint Surg Am 1998; 80: 910-22.

[30] Raut VV, Siney PD, Wroblewski BM. One-stage revision of infected total hip replacements with discharging sinuses. J Bone Joint Surg Br 1994; 76: 721-4.

[31] Raut VV, Orth MS, Orth MC. One stage revision arthroplasty of the hip for deep gram negative infection. Int Orthop 1996; 20: 12-4.

[32] Salvati EA, Chekofsky KM, Brause BD, Wilson PD Jr. Reimplantation in infection: a 12-year experience. Clin Orthop Relat Res 1982; 170: 62-75.

[33] Fitzgerald RH Jr. Infected total hip arthroplasty: diagnosis and treatment. J Am Acad Orthop Surgeons 1995; 3: 249-62.

[34] Springer BD, Lee GC, Osmon D. Systemic safety of high-dose antibiotic-loaded cement spacers after resection of an infected total knee arthroplasty. Clin Orthop Relat Res 2004; 427: 47-51.

[35] Buchholz HW, Elson RA, Engelbrecht E. Management of deep infection of total hip replacement. J Bone Joint Surg Br 1981; 63B(3): 342-53. 
[36] Ure KJ, Amstutz HC, Schmalzried TP. Direct-exchange arthroplasty for the treatment of infection after total hip replacement. J Bone Joint Surg Am 1998; 80(7): 961-8.

[37] Callaghan JJ, Katz RP, Johnston RC. One-stage revision surgery of the infected hip. Clin Orthop Relat Res 1999; (369): 139-43.

[38] Cierny G, DiPasquale D. Periprosthetic total joint infections. Clin Orthop 2002; 403: 23-8.

[39] Kilgus DJ, Howe DJ, Strang A. Results of periprosthetic hip and knee infections caused by resistant bacteria. Clin Orthop Relat Res 2002; 404: 116-24.

[40] Volin SJ, Hinrichs SH, Garvin KL. Two-stage reimplantation of the total joint infections: a comparison of resistant and non resistant organisms. Clin Orthop Relat Res 2004; 427: 97-100.

[41] Haddad FS, Muirhead-Allwood SK, Manktelow ARJ. Two-stage uncemented revision hip arthroplasty for infection. J Bone Joint Surg $\mathrm{Br}$ 2000; 82: 689-94.

[42] Lai KA, Shen WJ, Yang CY, Lin RM, Lin CJ, Jou IM. Two-stage cementless revision THR after infection. 5 recurrences in 40 cases followed 2.5-7 years. Acta Orthop Scand 1996; 67: 325-8.

[43] Weeden SH, Paprosky WG. Minimal 11-year follow-up of extensively porous-coated stems in femoral revision total hip arthroplasty. J Arthroplasty 2002; 17(4 Suppl 1): 134-7.
[44] Berry DJ, Chandler HP, Reilly DT. The use of bone allografts in two-stage reconstruction after failure of hip replacements due to infection. J Bone Joint Surg Am 1991; 73-A: 1460-68.

[45] Wang JW, Chen CE. Reimplantation of infected hip arthroplasties using bone allografts. Clin Orthop Relat Res 1997; 335: 202-10.

[46] Alexeeff M, Mahomed N, Morsi E, Garbuz D, Gross A. Structural allograft in two-stage revisions for failed septic hip arthroplasty. J Bone Joint Surg Br 1996; 78-B: 213-6.

[47] Müller M, Morawietz L, Hasart O, Strube P, Perka C, Tohtz S. Diagnosis of periprosthetic infection following total hip arthroplasty - evaluation of the diagnostic values of pre-and intraoperative parameters and the associated strategy to preoperatively select patients with a high probability of joint infection. J Orthop Surg 2008; 3: 31.

[48] Pelosi E, Baiocco C, Pennone M, et al. 99mTc-HMPAO-leukocyte scintigraphy in patients with symptomatic total hip or knee arthroplasty: Improved diagnostic accuracy by means of semiquantitative evaluation. J Nucl Med 2004; 45: 438-44.

[49] Larikka MJ, Ahonen AK, Junila JA, Niemelä O, Hämäläinen MM, Syrjälä HP. Extended combined $99 \mathrm{mTc}$-white blood cell and bone imaging improves the diagnostic accuracy in the detection of hip replacement infections: Eur J Nucl Med 2001; 28(3): 288-93.

(C) Pignatti et al.; Licensee Bentham Open.

This is an open access article licensed under the terms of the Creative Commons Attribution Non-Commercial License (http://creativecommons.org/licenses/by$\mathrm{nc} / 3.0 /$ ) which permits unrestricted, non-commercial use, distribution and reproduction in any medium, provided the work is properly cited. 